\title{
RF-Carpets that Compress Ions to High Density Beams
}

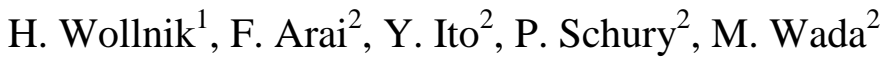 \\ ${ }^{1}$ Department Chemistry and Biochemistry, New Mexico State University, Las Cruces, NM, USA \\ ${ }^{2}$ SLOWRI Team, RIKEN, Nishina Center for Accelerator-Based Science, Wako-City, Saitama, Japan
}

\begin{abstract}
Ions that are moved by electric fields in gases follow quite exactly the electric field lines since these ions have substantially lost their kinetic energies in collisions with gas atoms or molecules and so carry no momenta. Shaping the electric fields appropriately the phase space such ion beams occupy can be reduced and correspondingly the ion density of beams be increased.
\end{abstract}

\section{Introduction}

Ion clouds can be shaped by electric fields only in so far as is allowed by Liouville's theorem [1], which states that the magnitude of the phase space occupied by an ion beam, which moves in vacuum, can not be changed. Ion beams that move in gases do not have this limitation, since they move substantially along electric field lines between collisions with gas atoms or molecules. However, ion clouds that move in gases increase their volumes over time by diffusion, so that the clouds' lateral and longitudinal dimensions increase with the square root of time. Consequently, ion beams cannot easily be focused to fine points, but wide ion beams can be transformed to narrow ones with increased ion densities.

In first experiments that proved this concept, clouds of charged microparticles were transported and compressed [2,3] by planar, cylindrical or conical electrode arrangements as shown in Figure 1, wherein to neighboring electrodes AC-potentials of typically $50 \mathrm{~Hz}$ were applied. Due to the corresponding ACfields the charged microparticles were pushed back and forth between neighboring electrodes without reaching either one of them. This is equivalent to an effective force $F_{\text {repel }}\left(Z_{C}\right)$ that repels the charged microparticles from the surface of the electrode arrangement and that decreases with the distance from this surface. In case the microparticles were pushed towards this surface by small forces $F_{\text {in }}$ caused by electric fields, gas flow forces or gravitation there is a distance $Z_{\text {hover }}$ at which the microparticles effectively hover above the surface of the electrode arrangement

Using planar or conical electrode arrangements whose surfaces are inclined by angles $\pm \alpha$ with respect to the substantially parallel incoming microparticles the mentioned force $F_{\text {in }}$ can be seen as being split into forces perpendicular and parallel to the surfaces of the electrode arrangement. In this case the microparticles hovered distances $Z_{\alpha}$ above these surfaces where $F_{\text {in }} \sin \alpha=F_{\text {repel }}\left(Z_{\alpha}\right)$ while the forces $F_{i n} \cos \alpha$ moved the microparticles parallel to these surfaces. Thus, the microparticles were concentrated to ensembles of higher particle densities, which was especially effective [3], when three-phase ACpotentials were applied to groups of three neighboring electrodes establishing a travelling wave field that moved the charged microparticles substantially parallel to the surface of the electrode arrangement.

Using AC-potentials of much higher frequencies the conical electrode arrangement of Figure 1 was also used to increase the densities in ion beams for mass spectrometric investigations [4]. In this case the conical electrode arrangement of Figure 1 was used, to which DC-potentials were superimposed over the RF-potentials between neighboring electrodes. Similarly as for the case of the charged microparticles the ions were driven back and forth between neighboring electrodes, effectively hovering above the surface 
of the conical electrode arrangement, while the DC-fields moved the hovering ions loss-free parallel to the cone surface and concentrated them to beams of small cross sections. Though such so-called ion funnels are very useful devices, it should be noted that they require high mechanical precision for their fabrication and for their alignment relative to the incoming ion beams.
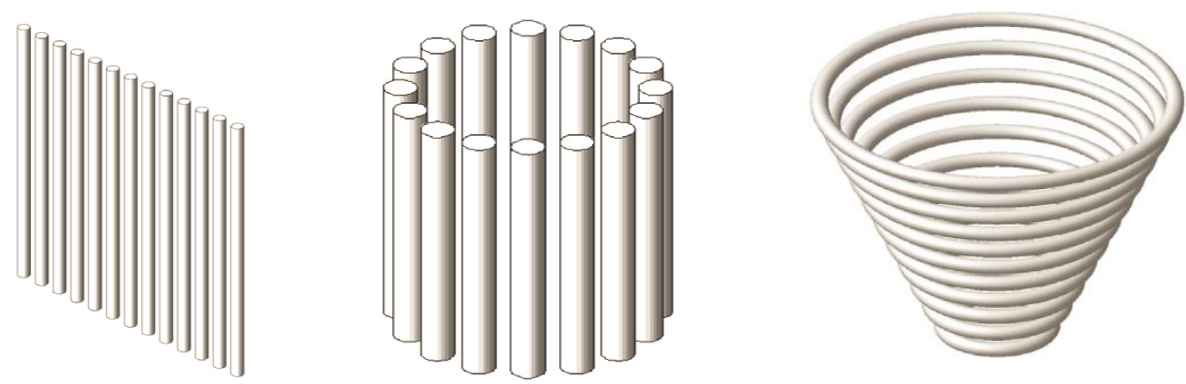

Figure 1. Electrode arrangements are shown, to neighboring electrodes of which alternating potentials were applied, so that the resulting AC-fields [2,3] effectively repel the ions from the electrode.

\section{Compression of clouds of slowed down high-energy ions}

A big improvement over the use of wire electrodes as shown in Figure 1 was to use $\mathrm{N}$ concentric ring electrodes formed as a conical or planar printed circuit board, a so-called RF-carpet [5,6] shown in Figure 2. Also in this electrode configuration high frequency fields are established between neighboring electrodes to which potentials $V_{R F}= \pm V_{00} \cos \omega_{00} t$ are applied, which cause the ions to be repelled from the surface of the RF-carpet and to hover above it, wherein $\omega_{00}$ was several MHz. The big advantage of a RF-carpet is that the electrode separations can be reduced and so the RF-fields be increased and that the shape of the electrodes can be formed and reproduced with high precision at low cost.

For singly charged ions the ion repelling force of the RF-carpet can be calculated [5,6] for every distance $Z_{C}$ above the RF-carpet by assuming that two neighboring electrodes are the electrodes of a quadrupole of aperture $2 r_{0}$ between which a potential difference $V_{R F}$ exists. The magnitude of this force is found as:

$$
F_{\text {repel }}\left(Z_{C}\right)=\frac{\sqrt{2} m \mu^{2} V_{R F}^{2}}{r_{0}^{3}}\left(1-\frac{Z_{C} \sqrt{2}}{r_{0}}\right)
$$

for $0 \leq Z_{C} \leq r_{0} / \sqrt{2}$, wherein $m$ and $\mu$ describe the mass and the mobility of an ion under consideration. Ions for which $F_{\text {repel }}\left(Z_{C}=0\right) \leq F_{\text {in }}$ will be pushed to the surface of the RF-carpet and be neutralized. All other ions will hover a distance of $Z_{\text {hover }}$ above the RF-carpet where $F_{\text {in }}=F_{\text {repel }}\left(Z_{\text {hover }}\right)$, if to the $\mathrm{n}^{\text {th }}$ of the $\mathrm{N}$ concentric electrodes a potential $V_{R F}=V_{00} \sin \left(\omega_{00}+n \pi\right) t$ is applied.

If additional electric fields are formed between neighboring electrodes, the hovering ions can be moved to an extraction orifice located at the center of the RF-carpet by DC-fields [6] or by fields of a travelling wave [7]. Consequently to the $\mathrm{n}^{\text {th }}$ carpet electrode one of the following potentials should be applied:

$$
\begin{gathered}
V_{D C}(n)=V_{R F} \cos \left(\omega_{00}+n \pi\right) t+V_{0} n \\
V_{A C}(n)=V_{R F} \cos \left(\omega_{00}+n \pi\right) t+V_{0} \cos \left(\omega_{0}+n \pi / 2\right)
\end{gathered}
$$


where $\omega_{00} \gg \omega_{0}$ and $n$ being the electrode number. In the case of Eq. (2) separate potentials must be provided to each ring electrode [5,6]. In the case of Eq. (3) only 4 potentials must be provided [7], since the potentials of the electrodes $n$ and $n+4$ are identical. This case is illustrated in Figure 2.

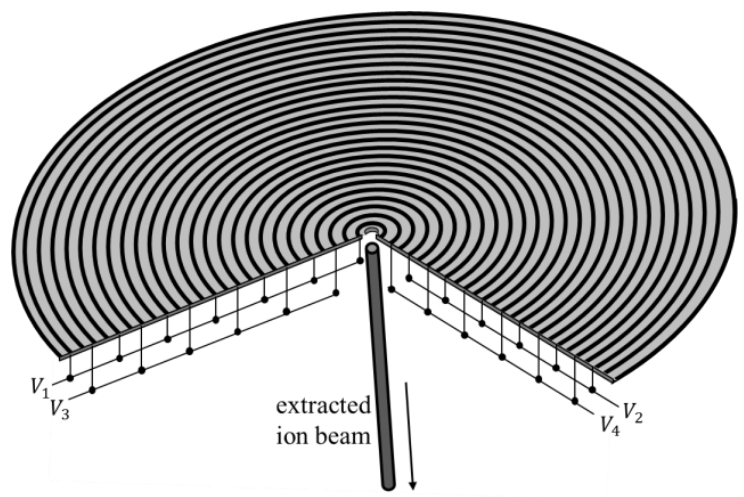

Figure 2. A printed-circuit RF-carpet is shown, wherein it is indicated that potentials according to Eq. (3) are applied to the $n^{\text {th }}$ ring electrode. Since in this case the potentials of the $n^{\text {th }}$ and the $(n+4)^{\text {th }}$ electrode are identical, only 4 potentials are required to form the surface repelling force due to the RFfield of frequency $\omega_{00}$ and the traveling wave field of frequency $\omega_{0}$.

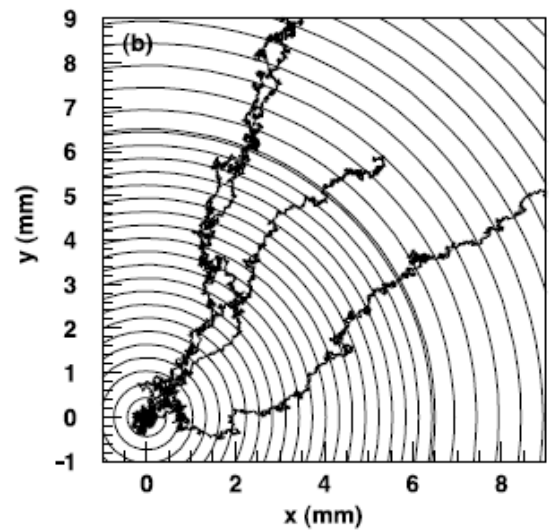

Figure 3. The motion of ions across the surface of a RF-carpet is shown as obtained from simulation calculations. Note here that the shown RF-carpet has two regions of concentric ring electrodes.

By both methods the ions are pushed towards the extraction orifice of the RF-carpet, wherein the trajectories differ individually due to collisions with gas atoms or molecules. An illustration of how ions move over the surface of the RF-carpet is illustrated in Figure 3 for which the trajectories have been obtained from numerical simulation calculations. Due to the statistical nature of the ion motion there is a non-vanishing probability that some of the ions reach the surface of the printed circuit where they are neutralized. This probability is reduced if $Z_{\text {hover }}$ is increased, which requires an increased force $F_{\text {repel }}\left(Z_{C}\right)$, which can be achieved (see Eq. (1)) by increasing $V_{R F}$ or by building the RF-carpet with a reduced $\sqrt{2} r_{0}$ separation between neighboring electrodes [8]. So far RF-carpets have mainly been used to concentrate energetic heavy-ion reaction products for further analyses $[5,6,8]$. This was achieved in large gas-filled vessels that contain RF-carpets (see Figure 4). Such vessels are typically filled with He gas at a pressure of $\approx 100$ mbar, which does not have a big stopping power, but keeps ions charged because of the high ionization energy of He. 
However, there are also $\mathrm{He}^{+}$ions formed in large quantities by knock on events during the slowing down process of the energetic nuclei. The large number of these $\mathrm{He}^{+}$ions caused severe space-charge forces [6] in the gas-filled vessel. Fortunately these $\mathrm{He}^{+}$ions do not contribute to the extracted ion beam, because they have a very low mobility $\mu$ when moving in He and thus experience only a very small repelling force $F_{\text {repel }}\left(Z_{C}=0\right)$, which is always smaller than the force $F_{\text {in }}=q E_{Z}$. Thus all these $\mathrm{He}^{+}$ ions reach the RF-carpet, where they are neutralized.

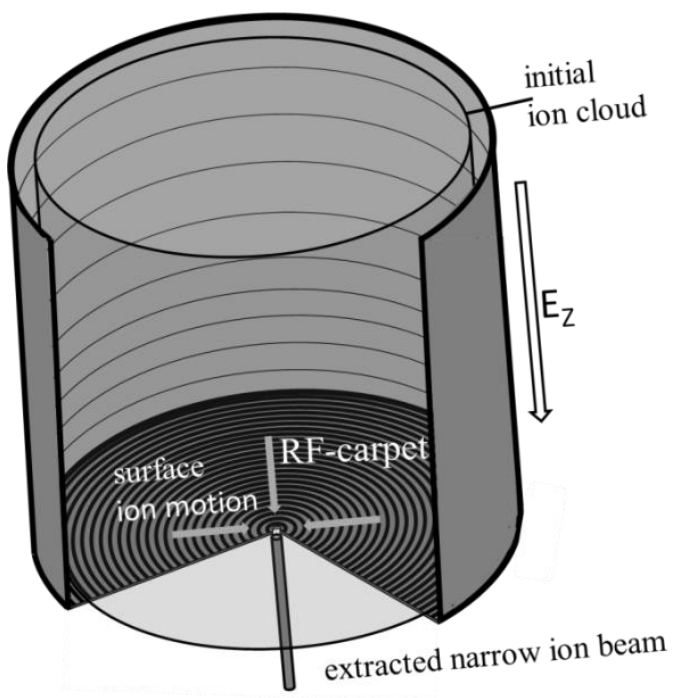

Figure 4 A retardation chamber of $\approx 1 \mathrm{~m}$ in length and $\approx 0.3 \mathrm{~m}$ in diameter is shown, that is filled with He-gas, in which energetic nuclei are slowed down, while keeping their charge. These ions are pushed by the electric field $E_{Z}$ towards the planar RF-carpet of concentric ring electrodes. Because the force $q E_{Z}$ on the ions is counterbalanced by the $F_{\text {repel }}\left(Z_{C}\right)$ of Eq. (1), caused by the RF-field between the ring electrodes, the ions hover a short distance above the RF-carpet, while additional electric DC- or ACfields drive the ions towards the center orifice, through which they can be extracted.

For atomic ions the ion mobility $\mu$ is quite accurately proportional to the inverse of the gas pressure $p$ in the gas stopper sketched in Figure 4, showing that $F_{\text {repel }}\left(Z_{C}\right)$ increases for a reduced pressure $p$. For molecular ions this relation is more complex, though also in this case a reduction of $p$ will increase $F_{\text {repel }}\left(Z_{C}\right)$. This is the reason that ion funnels [4] as well as RF-carpets [5,6] work best at reduced pressures. However, since energetic ions are stopped better in a gas of higher pressure in most cases $p$ is increased as much as possible, which requires to increase $V_{R F}$ in Eq. (1) and even more importantly reduce $\sqrt{2} r_{0}$, the distance between neighboring ring electrodes on the RF-carpet. The smallest electrode separation that has been reached so far was $\sqrt{2} r_{0}=0.16 \mathrm{~mm}$ [8], which allowed to increase the He pressure to $\approx 300$ mbar and still extract $100 \%$ of all ions stopped in the gas vessel of Figure 4 , while the voltage $V_{R F}$ still stayed well below the discharge limit.

\section{Compression of beams of low energy molecular ions and their mass and mobility analysis}

Also atomic or molecular ion beams, formed in ion sources can be compressed as long as they move in a gas atmosphere and so be transformed to beams of higher ion densities. Commonly the ions in such beams are extracted from high pressure ion sources by electric fields of about $100 \mathrm{~V} / \mathrm{mm}$ as is the case for instance in an electrospray ion source. Using such fields to push the ions towards a flat RF-carpet can easily cause forces $F_{\text {in }}$ that push the ions too strongly towards the RF-carpet, so that they can reach the 
RF-carpet surface. To limit these forces the electric field close to the RF-carpet can be reduced to tolerable values and the ions be embedded in a gas stream that pushes them towards the RF-carpet. This technique has been used successfully [9] to achieve narrow beams of molecular ions and to inject them into a multi-reflection time-of-flight mass spectrograph $[10,11]$ where they were analyzed with mass resolving powers $m / \Delta m \geq 100,000$. The overall experimental setup for this experiment is illustrated in Figure 5 showing:

1. that ions formed in an electrospray ion source were extracted in air at a pressure of $1 \mathrm{~atm}$ and

2. that the extracted ions were guided towards a capillary of inner diameter of $0.5 \mathrm{~mm}$. Ions that arrived at the entrance of the capillary within a volume of about $2 \mathrm{~mm}^{3}$ were sucked into the capillary together with the gas in which they were embedded.

3. The gas-transported ions were blown into a vacuum of $\approx 10$ mbar towards a RF-carpet, which guided them to an extraction orifice of $0.5 \mathrm{~mm}$ diameter, from where they were accelerated by $\approx 100 \mathrm{eV}$ so that

4. they could be transported by a quadrupole beam guide to a multi-reflection time-of-flight mass analyzer in a good vacuum of $\leq 1$ nbar.

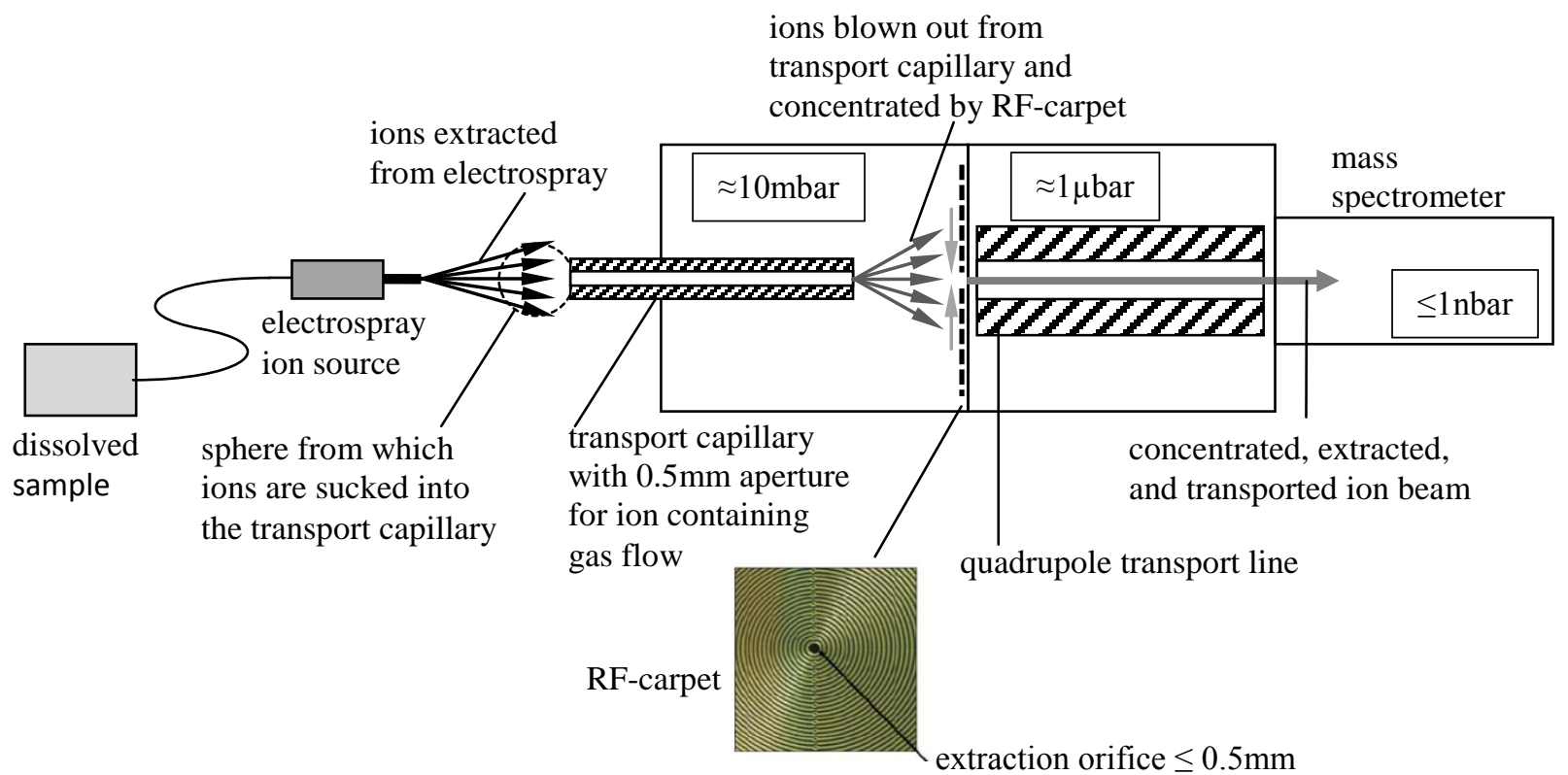

Figure 5. Shown is how ions formed in an electrospray ion source were guided towards a capillary into which a gas volume of a few cubic millimeters is sucked through a capillary into a vacuum of $\approx 10 \mathrm{mbar}$ together with the embedded ions. An RF-carpet then guided the ions to an extraction orifice from where they could be extracted as a beam of $\leq 0.5 \mathrm{~mm}$ diameter, which then was transported by a quadrupole beam guide from a vacuum of $\approx 1 \mu$ bar to a mass analyzer at $\approx 1$ nbar.

A good mass analysis is most important for the investigation of small and large molecules. However, this mass analysis can only provide information of the composition of the molecule under investigation. There is no information of the shape of this molecule and the arrangement of subparts of it. This can only be obtained by an ion mobility analysis. Commonly such an analysis requires a separate investigation that employs a specific mobility analyzer. However, the here described RF-carpet can already act as such a mobility analyzer or mobility selector. Ions that are moved to the central extraction orifice by a travelling wave must move through the gas with the same speed as the travelling wave, 
which is an efficient transport mechanism only for ions of a specific mobility [12]. The mobility resolving power, however, is limited, since the ions have travelled different distances to reach the extraction orifice depending on the position at which they have reached the RF-carpet. To improve this mobility selection process it can be divided into two phases.

1. In a first phase the travelling wave of Eq. (3) is shaped so that the ions move to the outer rim of the RF-carpet where they are accumulated in a ring-like potential well formed by the potentials of the outermost ring electrodes.

2. In a second phase the travelling wave of Eq. (3) is shaped so that the accumulated ions are driven towards the extraction orifice with a selected speed. Similarly to the T-wave technology [12] this transport is mobility selective because of the speed of the travelling wave.

This two phase transport could also be achieved by the fields of Eq. (2) in which case even a mobility spectrum could be recorded directly. This would require, however, that each ring electrode of the RFcarpet has a separate contact, which -as mentioned above- requires a complex printed circuit design.

\section{Conclusions}

Ion beams that move in a gas atmosphere are not subject to Liouville's theorem allowing to compress the phase space that such ion beams occupy. This provides possibilities to improve the quality of beams of ions that are to be investigated for instance in mass spectrometers or in accelerators by reducing the diameter of an ion beam and so increase the ion density for instance by the use of an RF-carpet. Described is also that the ion transport across an RF-carpet can used to achieve a mobility spectrum or to alternatively select ions of a specific mobility, when proper AC-potentials are applied to the ring electrodes that form a travelling-wave field, which transports hovering ions to the carpet center.

\section{References:}

[1] H Wollnik in "Optics of Charged Particles", 1987, (Acad. Press, Orlando).

[2] S Masuda et al, Int. Staubtagung, Staub-Reinhaltg Luft (1970), p. 449.

[3] S Masuda et al, Electr. Eng. Jpn. 92 (1972), p. 9.

[4] RT Kelly et al, Mass Spectrom. Rev. 29 (2010), p. 294.

[5] M Wada et al, Nucl. Instrum. Meth. B 204 (2003), p. 570.

[6] A Takamine et al, Rev. Sci. Instrum. 76 (2005), 103503.

[7] G Bollen, Int. J. Mass Spectrom. 299 (2011), p. 131.

[8] F Arai et al, Int. J. Mass Spectrom. 362 (2014), p. 56.

[9] S Naimi et al, Int. J. Mass Spectrom. 337 (2013), p. 24.

[10] H Wollnik and M Przewloka, Int. J. Mass Spectrom. 96 (1990), p. 267.

[11] P Schury et al, Nucl. Instrum. Meth. B 335 (2014), p. 39.

[12] AA Shvartsburg and RD Smith, Anal. Chem. 80 (2008), p. 9689. 